\title{
The Fastest Simulation of Protein Folding Based on Torsion Angles
}

\author{
Seonghoon Jeong ${ }^{1}$ \\ ${ }^{1}$ Roio synthetic biology
}

October 8, 2020

\begin{abstract}
Backgrounds: Enormous number of possible conformations in the protein structure simulation have led molecular dynamics researchers to be frustrated until now. Some methods with defects ended their experiments into failure. This made them fail to determine the structure and function of folded protein in stable state with the lowest potential energy. This apparently exist in nature. The purpose of resolving a protein folding pathway that follows protein backbone residues torsional inertia was accomplished. Results: A new method, torsion angle modeling, was adopted focused on the rotation of dihedral angles. The potential energy was calculated by rotating torsion angles of the peptide with 8 residues. It was found that when moving in the order of torsional inertia, 8 residues swivel in sequence. Six passes were repeated to find the lowest value. Conclusion: The protein backbone torsion angle plays very important role in predicting protein structure. Actually it was thousand times faster or more than others to get the obvious pathway.
\end{abstract}

\section{Introduction}

Simulation of protein structure has been the most important in bioinformatics. In the methods of prediction, there are homology modeling, threading, and ab initio modeling. Ab initio starts without any results from experiment. So this is more useful than any others to look into the structure of the unknown protein. This constructs it's structure by physical methods to the bare atoms of the protein. But this involves the defects of inaccuracy and slowness than others in prediction(Jung, 2013).

Developing an algorithm for ab initio modelling to simulate protein structure is very important(K.A. Dill and J.L. MacCallum, 2012). This has two types of algorithms; one is molecular dynamics(MD) and the other is Monte Carlo(MC). This randomizes the location of atoms to predict protein structure in 3D space. That sets force fields to estimate atom positions at every moment.

Exponential time has spent to calculate all of the unknown protein's conformations by using the highest capacity computer with MC and MD. Note that the structure of the protein changes between microseconds, and furthermore, the positions of the atoms in this structure occur in shorter pico and femto seconds. Thus, to obtain stable form of the molecule is still difficult for researchers.

In nature protein folds faster and more naturally than this. Namely, in vivo folding is faster than in vitro.(Nicola et al., 1999; Kolb et al., 2000; Cabrita et al., 2010; O’Brien et al., 2011, Fedorov and Baldwin, 1999; Seckler et al., 1989) So Levinthal addressed paradoxically that there is the apparent pathway in the folding(J. T. P. DeBrunner and E. Munck, 1969). This is true. And Karplus suggested that the 'bias towards the native state' over much of the effective energy surface may govern the folding process(M. Karplus, 1997). We appreciate this is the pathway of folding that occurs in protein synthesis from mRNA transcript.

Computational simulation of this cotranslational folding based on the numerical evidences appeared in 1995(Law, 2017; de Oliveira et al., 2015; Ellis et al. 2010; Lu and Liang, 2008; Srinivasan and Rose, 1995; Alexandrov, 1993). Besides, we introduce many experimental evidences and opinions of researchers for 
protein folding during intracellular gene translation(Cymer, F. and von Hijne, G., 2013; Fedorov, A.N., and Baldwin, T.O., 1997).

It is worth to look into cotranslational folding, because there are differences between simulated and experimental structures. We derived the structures from cotranslational and torsional algorithm. Cotranslational folding simulation was managed before by using SAINT algorithm(Ellis et al., 2010). 3D molecular dynamics was used in this experiment. All movements in the Cartesian space were considered as possible in most molecular dynamics algorithms by most of the researchers. These employ strong covalent bond stretching and bond angle bending terms in their force fields. Though covalent bond stretching and bond angle bending are affected by the force field, these model's description abandons the fact that only rotations about covalent single bond occurs.

We used, thus, backbone torsion angle as the only degree of freedom to describe the motion of protein folding. These models might hamper to interpret the mechanism of protein folding. And helpful manual modification is restricted from researchers. These difficulties could be settled by torsion angle method. Also this is more realistic than other 3D models full of lattice approximations.

\section{Materials and Methods}

The polypeptide is free swivel chain and has levers which collide to water molecules. The collision in the protein folding in the cell follows the Brownian movement. It is much stronger. The calculation of the translational molecular kinetics is based on the translational enthalpy data. Thus, the kinetic energy of the water molecule is,

$$
\sim 2.0(\mathrm{kcal} / \mathrm{mol})=\frac{1}{2} m v^{2}
$$

And the velocity of water molecule gives,

$$
\begin{gathered}
2.0(\mathrm{kcal} / \mathrm{mol})=\frac{1}{2} \times 18(\mathrm{~g} / \mathrm{mol}) \times v^{2} \\
v^{2}=20(\mathrm{kcal} / \mathrm{g})
\end{gathered}
$$

1 calory is $4.1868 \mathrm{~J}(1 \mathrm{cal}=4.1868 \mathrm{~J})$, so

$$
\begin{gathered}
v^{2}=20 \times 4.1868 \times 10^{3}(\mathrm{~J} / \mathrm{g}) \\
=20 \times 4.1868 \times 10^{3}(\mathrm{~N} \bullet \mathrm{m} / \mathrm{g}) \\
=20 \times 4.1868 \times 10^{3}\left(\mathrm{~kg} \bullet \mathrm{m} / \mathrm{s}^{2} \bullet \mathrm{m} / \mathrm{g}\right) \\
=20 \times 4.1868 \times 10^{6}\left(\mathrm{~m}^{2} / \mathrm{s}^{2}\right)
\end{gathered}
$$

Hence, velocity of water molecule is

$$
\begin{gathered}
v=\sqrt{20 \times 4.1868} \times 10^{3}(\mathrm{~m} / \mathrm{s}) \\
=\sqrt{83.736} \times 10^{3}(\mathrm{~m} / \mathrm{s}) \\
=9.151 \times 10^{3}(\mathrm{~m} / \mathrm{s})
\end{gathered}
$$


This velocity is fast enough to rotate backbone's residue with torques(Serway, R. A. and Jewett, Jr. J.W., 2003; F. H. Stillinger, 1975).

Torsional inertia in the swivels are different for each amino acid. In a backbone, only one residue rotates, the others do not. While the former has small inertia, the latter has larger one. The difference of torsional inertia comes from the type and number of atoms and the length of side chain, and the mass and length of the backbone on both sides of the residue. The middle residue has a small rotational inertia, because the rotational inertia is proportional to the square of the distance.

The following(Figure 1) represents only two residues among many in the backbone. Among these connected residues, only two residues with the least rotational inertia were shown. Between them, A has smaller rotational inertia than B. When water molecules collide, A rotates first. B is a residue that stops while residue A rotates, and then returns as soon as A stops.

Residue $\mathrm{A}$, has rotational momentum $\mathrm{P}_{\mathrm{a}}$ is,

$P_{A}=m_{A} R_{A}^{2} \omega_{A}$

$I_{A}=m_{A} R_{A}^{2}$

If $\mathrm{B}$ rotates, the rotation momentum $\mathrm{P}_{\mathrm{b}}$ of $\mathrm{B}$ is,

$$
P_{B}=m_{B} R_{B}^{2} \omega_{B}-P_{A}
$$

When $\mathrm{A}$ does not rotates, the rotation momentum $\mathrm{P}_{\mathrm{b}}$ of $\mathrm{B}$ is

$P_{B}=m_{B} R_{B}^{2} \omega_{B}$

If $\mathrm{A}$ rotates, the rotation momentum $\mathrm{P}_{\mathrm{b}}$ of $\mathrm{B}$ is

$P_{B}=m_{B} R_{B}^{2} \omega_{B}-P_{A}$

If $\mathrm{A}$ stops rotation, $\mathrm{P}_{\mathrm{a}}$ becomes zero and $\mathrm{P}_{\mathrm{b}}$ is not reduced. So $\mathrm{B}$ can rotate more easily. If $\mathrm{A}$ rotates, $\mathrm{P}_{\mathrm{a}}$ becomes larger and $\mathrm{P}_{\mathrm{b}}$ is strongly reduced. Therefore, $\mathrm{B}$ cannot rotate easily.

In figure 2, the angle between the axel with $\mathrm{A}$ residue and B1 is $104.5^{\circ}$. Assuming that B rotates while A also rotates, residue A must handle this rotational movement of residue B. However, if it is $90^{\circ}$, because the radius of rotation $\left(R=1 \times R_{0}\right)$ is the longest, the rotational inertia of residue $B$ is affected to residue $A$. Since, residue A can hardly turn without the strongest force because rotational inertia $\left(\mathrm{I}=\mathrm{R}_{2} \times \mathrm{m}\right)$ is the maximum.

To receive the half of the magnitude of the force mentioned above, it must be $135^{\circ}$. The radius of $\mathrm{B}_{2}$ is,

$$
R=\cos 45 \times R_{0}=\frac{1}{\sqrt{2}} R_{0}
$$

And inertia I is,

$$
I=m R^{2}=\frac{m R_{0}^{2}}{2}=\frac{1}{2} I_{0}
$$

The bond angle between the two residues $\mathrm{A}$ and $\mathrm{B}$ is $104.5^{\circ}$. The inertia of $\mathrm{B}_{2}$ at $104.5^{\circ}$ is,

$$
I=\left(R_{0} \times \cos (14.5)\right)^{2} \times m=0.97^{2} \times I_{0}=0.94 \times I_{0}
$$

This is almost the same as the value of $90^{\circ}$ with the most difficulty. In conclusion, residue $\mathrm{B}_{2}$ cannot rotate with $\mathrm{B}_{1}$ at the same time. 
In short, all of the above are summarized as follows. If there are many residues of different sizes, they do not rotate at the same time. The residue with the smallest inertia rotates first. As soon as it stops rotating, the residue with the next smallest inertia turns. When stops rotating, the next residue with more inertia than this rotates. In the case of multiple residues, the rotational inertia is ordered from the smallest.

The coordinates of short polypeptide with important points above was set. And it was compared with the structure from solution NMR. The cotranslational folding and torsional movement of atoms which are different from others was proved. The most representative model of each NMR assay was compared with the structure from the new folding algorithm. Typical structure alignment algorithms including TM-align(Y. Zhang and J. Skolnick, 2005) were not used in the comparison. These algorithms moves frame with insertions and deletions even in the case of the comparison of identical amino acid sequence. The result of the comparison was represented by $\log \operatorname{Pr}$ and RamRMSD(S. Jung, et al., 2011). And it was illustrated in the graph of torsion angle along the residue number(Figure 3). The change of the potential energy during the initial folding and following optimization is also displayed in the graph(Figure 4).

\section{Data set}

A single asymmetric chain structure was adopted in the PDB archive on the condition that the length of the chain was 8 , the sequence identity was $90 \%$ or less, and that there were no heteroatoms. Structures which only contains protein without nucleic acids of DNA, RNA, or DNA-RNA hybrid was selected. Two structures of $1 \mathrm{n} 9 \mathrm{v}$ and 1 oeh was set. And $10 e \mathrm{~h}$ was abandoned because it was fragmented. Finally, $1 \mathrm{n} 9 \mathrm{v}$ which is the angiotensin peptide was used in this work. 'DRVYIHPF' is the amino acid sequence of $1 \mathrm{n} 9 \mathrm{v}$.

\section{Cotranslation Folding of Initial Structure}

Cotranslational folding was performed with ProtTorter using torsion angles. Whenever a new amino acid was added, the potential energy was calculated, considering every conformation following the change of and $\psi$ angles. As peptide bonds revolve around the backbone, we supposed they move 1 degree by 1 degree. So, 360 cases were observed. Local minima and the global minimum was gotten. These were to predict the initial structure of angiotensin.

\section{Iterative Optimizations}

This simulation became accurate by the peptide collision with water molecules, residues received larger torques, and planar amino acids to rotate faster. The length of the side chain was estimated as the maximum of bonds from the $\mathrm{C} \alpha$ atom. The order of calculation of the pair of bonds of the residue from the sum of the priority from torsional property.

The 8 amino acids of $1 \mathrm{n} 9 \mathrm{v}$ were arranged into ' $\mathrm{D}(7) \mathrm{R}(2) \mathrm{V}(8) \mathrm{Y}(1) \mathrm{I}(5) \mathrm{H}(4) \mathrm{P}(6) \mathrm{F}(3)$ '. The smaller number was calculated first and received higher priority.

Consequently, the $4^{\text {th }}$ peptide bond between $\operatorname{Tyr}(\mathrm{Y})$ and Ile(I) was first calculated $(1+5=6)$, and the bond between $\operatorname{Tyr}(\mathrm{Y})$ and $\operatorname{Val}(\mathrm{V})(1+8=9)$. The order of priority is ' $\mathrm{D}-(3)-\mathrm{R}-(4)-\mathrm{V}-(2)-\mathrm{Y}-(1)-\mathrm{I}-(6)-\mathrm{H}-(7)-\mathrm{P}-(5)-\mathrm{F}$ '. The numbers in the round brackets designate the order of the linkage among residues. The dihedral angles flanking peptide bond are $\varphi$ and $\psi$ angles. Between these two dihedral angles, we calculated the one close to the higher priority first, and the other close to the lower priority later.

In this order, we iteratively optimized the initial structure from cotranslational folding. This was performed until the convergence of potential energy. The structure in which potential energy converged in six times from the initial structure was taken.

\section{Results and Discussion}

Angiotensin was simulated using ProtTorter(Jung, 2013). While other simulation programs are invisible and untouchable, this can check each structure in every step. This can directly manipulate. Energetically stable local minima structure which is still smaller than the reference structure was observed. 
The structure of $1 \mathrm{n} 9 \mathrm{v}$ from the simulation with ProtTorter is shown in figure 5. Loop structure was found in most of the 8 residues except the first aspartate residue in the angiotensin peptide. The dihedral angles( , $\psi$ ) were usually around the range of $\left(25^{\mathrm{O}}-30^{\mathrm{O}}, 0^{\mathrm{O}}-5^{\mathrm{O}}\right)$. We can see the vivid difference between this and other simulations (Figure 3). Experimental structure regularly oscillated above and below the $0^{\circ}$, while simulated angles were positive in the N-terminal region. These deviated far from the C-terminal region.

After simulating, we obtained angles and local energy minima. And all of these were arranged into table 1 for clarity. The initial and six iterations were arrayed in the row of the table and seven bonds in the column. In the end, 49 arguments were placed in the table. The $\psi$ angle is written on the top of the cell and the $\varphi$ in the bottom. In each cell, angles and the number of local minima are written.

The structure was simulated from very large search space. As table 1 shows, initial structure of cotranslational folding was from about 52 conformations. For this peptide composed with 8 residues, the most stable structure was from usually about 70-90 structures. It is very efficient to compare with typical molecular dynamics.

$\log P r$ value(Table 2) signifies the difference of the two compared structures with more weight on the more closer similarity. There are eight dihedral angles each for residue from 1 to 8 in both reference and simulated structures. When the two angles of the same residue is very similar, those values were made to be equivalent. On the contrary, they were made to be different.

The $\log P r$ values of simulated structures increased toward the later iterations implying the convergence in optimizations. The lowest $\log \operatorname{Pr}$ value of -15.18 was observed from the pair of $5^{\text {th }}$ and $6^{\text {th }}$ optimization. The fact that lower $\log \mathrm{Pr}$ values in the pairs of nearer iterations than the farther ones was found. The highest $\log P r$ value among the pairs of adjacent passes was -4.62 in init. and opt. 1(Table 2). There were difference between the cotranslational path and the torsional one.

RamRMSD(Table 2) is the RMS(root mean square) deviation between the positions of residues on the Ramachandran plot(Ramakrishinan and Ramachandan, 1965). RamRMSD is similar to logPr. This includes the growing similarity among later iterations. Pairs in closer passes had lower RamRMSD values than farther passes. The highest RamRMSD among the pairs of adjacent passes was 47.17. This was calculated from the pair between the initial and the first optimization pass.

In figure 4, the change of energy in the folding of initial structure and in the optimization were illustrated. The potential energy drastically fluctuated in the simulation of initial structure in cotranslational folding. This partly indicates that addition of amino acid is either favorable or unfavorable in each different circumstance. This fluctuation is different from following iterations. This reflects the strong effect of the change of configurations. During the six passes of optimizations, the potential energy decreased saltatorily. This shows that there are a few critical bonds which strongly influence the potential energy of the whole molecule. Demonstrating the fast convergence of the algorithm to the global energy minima, the potential energy remained as being conserved after three passes of optimizations.

Comparing this simulation and others' experimental structure of $1 \mathrm{n} 9 \mathrm{v}$ with RamRMSD and $\log P r$, this is more stable than others by global minimum of $-1.704(\mathrm{kcal} / \mathrm{mol})$. The most correlation between each generated structure from its initial structure with adjacent passes have displayed. This increased for the later rounds of iterations. During the folding simulation, the energy dropped saltatorily(Figure 4).

The structure from NMR spectroscopy was very different from this simulation. The average of all logPr values ranged from -0.80 to -1.01 . RamRMSD varied from 116.68 to 136.28 . Although the simulated structure is somewhat different from the reference experiment, it is quite appreciable regarding the low and negative potential energy of $-1.704(\mathrm{kcal} / \mathrm{mol})$. This negative potential energy remark that this structure is stable in the vacuum environment. This structure is not only a low and stable energy structure but also a possible actual energy minimum because it is an energy minimum along the torsional propensity path. The torsional propensity path must be the path from Levinthal paradox.

Results suggest five parts to be discussed as follows. First, the difference of structure was due to the 
electrostatic interaction of atoms and the torsional barrier of rotatable bonds. Given motive force, a stronger turn is induced. This shortens the length of the loop structure. The structure of $\alpha$-helix was observed from lattice model without the consideration of any detailed electrostatic or torsional potential energy(Leach, 2001). Thus, additional restraints of non-electrostatic interaction would induce the current loop structure into well-known helices.

Second, another reason for the difference is the utilized force field. There was difference between NMR spectroscopy and this simulation. It is because that was conducted within an aqueous solution and this was performed under the vacuum environment. The difference between the simulated and the NMR structures was brought by the neglect of the interaction of solvents with the protein molecule. And hydrophobic effect and free energy from solvent accessible surface area could be obtained from experiments. This could be applied to structure simulation.

Third, it is very interesting for its fast convergence of the iterations. Although there is a false convergence, It is quite fast finding converging structure in 6 passes. Converging energy minima were quickly obtained following this method.

Fourth, simplified representation of the three dimensional structure of a protein in torsional system was applied. This regenerates the movements of atoms of polypeptide chain in the cellular environment. The fundamental characters of ribosome bound cotranslational folding could be generated. Three dimensional information can be transformed into one dimension by computing easily. This could be operated with sequence alignment algorithms in personal computer fast and correctly as BLAST(Altshul, 1990).

Fifth, ProtTorter adopted torsional representation of atomic movements(Jung, 2013). The results showed fast convergence to the stable form and which was negative and big in the potential energy. However, this path should be solidly validated referring longer polypeptide chains and larger numbers of test proteins. The structure from this program is different from that of the representative NMR in torsion angle. This occurred in folding pathway or force field.

\section{Conclusion}

Initial structure formation corresponded cotranslational protein folding. Torsion angels of the residue on backbone rotates one by one following torsional inertia. Optimization based on torsional inertia has reduced the number of candidate structures for the native structure. Stable conformation with low potential energy was obtained after input angiotensin sequence into ProtTorter which has the functions of torsion angle rotations and potential energy calculation. Coordinates of local minima to the stable structure were observed in very short time. The clearest folding pathway was represented in the most stable structure coordinate.

\section{Reference}

Altschul SF, Gish W, Miller W, Myers EW, Lipman DJ. Basic local alignment search tool. J Mol Biol. 1990;215(3):403-10.

Cymer F, von Heijne G. Cotranslational folding of membrane proteins probed by arrest-peptide-mediated force measurements. Proc Natl Acad Sci USA. 2013;110(36): 14640-5.

De Oliveira SHP, Shi J, Deane CM. Building a better fragment library for de novo protein structure prediction. PLoS One. 2015;10(4):e0123998.

Dill KA, MacCallum JL. The protein-folding problem, 50 years on. Science. 2012;338:1042-1046.

Ellis JJ, Huard FPE, Deane CM, Srivastava S, Wood GR. Directionality in protein fold prediction. BMC Bioinformatics. 2010;11:172.

Fedorov AN, Baldwin TO. Co-translational protein folding. J Biol Chem. 1997;272:32715-32718.

Jung S(Seonghoon Jeong old name), Chapter I. Introduction. In: Development of globularity and torsion based analysis methods for protein structures. Seoul National University; 2013. p. 8-19. 
Jung S(Seonghoon Jeong old name), Chapter V. ProtTorter: A protein structure modeler with torsion angle system. In: Development of globularity and torsion based analysis methods for protein structures. Seoul National University; 2013. p. 86-113.

Jung S, Bae S, Son HS. Validity of protein structure alignment method based on backbone torsion angles. J Proteom Bioinform. 2011;4:10.

Karplus M, The levinthal paradox: yesterday and today. Fold Des. 1997;2:S69-S75.

Law E. Computational studies of structural motifs and cotranslational folding mechanisms in membrane and soluble proteins. Oxford University; 2017.

Leach AR. Molecular modeling: principles and applications. 2nd ed. Prentice Hall; 2001. p.513, 516, 519.

Levinthal C. Mössbauer spectroscopy in biological systems proceedings. Univ Illinois Bull. 1969;67(41):22-24.

Ramakrishnan C, Ramachandran GN. Stereochemical criteria for polypeptide and protein chain conformations, II. Allowed conformations for a pair of peptide units. Biophys J. 1965;5(6): 909-933.

Serway RA, Jewett Jr JW. Chapter 10. Rotation of a rigid object about a fixed axis. In: Physics for scientists and engineers. 10th ed. Brooks Cole; 2003. p. 292-335.

Stillinger FH. Theory and molecular models for water. In: Prigogine I, Rice SA, editors. Advances in chemical physics, volume XXXI, John Wiley \& Sons; 1975. p. 68.

Zhang Y, Skolnick J. TM-align: A protein structure alignment algorithm based on the TM-score. Nucl Acids Res. 2005;33:2302-2309.

Table 1. Backbone Dihedral Angle and Number of Energy Minima

\begin{tabular}{llllllll}
\hline & init. + & opt. 1++ & opt. 2 & opt. 3 & opt. 4 & opt. 5 & opt. 6 \\
\hline $1^{\text {st }}$ bond & ang. $(\min . \#)$ & & & & & & \\
$\Psi$ & $-171(4)$ & $-171(4)$ & $-171(4)$ & $-171(4)$ & $-171(4)$ & $-171(4)$ & $-171(4)$ \\
$\Phi$ & $26(4)$ & $27(4)$ & $26(4)$ & $26(4)$ & $26(4)$ & $26(4)$ & $26(4)$ \\
$2^{\text {nd }}$ bond & & & & & & & \\
$\Psi$ & $2(2)$ & $1(5)$ & $5(5)$ & $5(5)$ & $5(5)$ & $5(5)$ & $5(5)$ \\
$\Phi$ & $35(5)$ & $31(6)$ & $29(5)$ & $30(6)$ & $30(6)$ & $30(6)$ & $30(6)$ \\
$3^{\text {rd }}$ bond & & & & & & & \\
$\Psi$ & $1(2)$ & $0(10)$ & $2(9)$ & $1(8)$ & $1(8)$ & $1(8)$ & $1(8)$ \\
$\Phi$ & $31(4)$ & $27(9)$ & $29(9)$ & $26(8)$ & $26(8)$ & $26(8)$ & $26(8)$ \\
$4^{\text {th }}$ bond & & & & & & & \\
$\Psi$ & $1(2)$ & $2(6)$ & $0(8)$ & $4(8)$ & $3(7)$ & $3(7)$ & $3(9)$ \\
$\Phi$ & $-150(7)$ & $-39(10)$ & $-39(15)$ & $-35(12)$ & $-34(11)$ & $-34(11)$ & $-34(10)$ \\
$5^{\text {th }}$ bond & & & & & & & \\
$\Psi$ & $-153(12)$ & $-24(5)$ & $-1(6)$ & $-179(11)$ & $-179(11)$ & $180(11)$ & $180(11)$ \\
$\Phi$ & $-179(8)$ & $-169(5)$ & $30(7)$ & $-166(5)$ & $-167(11)$ & $-169(11)$ & $-170(11)$ \\
$6^{\text {th }}$ bond & & & & & & & \\
$\Psi$ & $4(3)$ & $-154(8)$ & $11(6)$ & $-176(8)$ & $-175(8)$ & $-174(8)$ & $-174(8)$ \\
$\Phi$ & $-28(4)$ & $-67(5)$ & $-41(9)$ & $-69(5)$ & $-68(5)$ & $-68(5)$ & $-68(5)$ \\
$7^{\text {th }}$ bond & & & & & & & \\
$\Psi$ & $-176(3)$ & $180(3)$ & $179(3)$ & $6(5)$ & $6(3)$ & $6(3)$ & $6(3)$ \\
$\Phi$ & $168(5)$ & $168(5)$ & $171(4)$ & $34(5)$ & $35(8)$ & $35(8)$ & $35(7)$ \\
Search Space & 52 & 72 & 88 & 81 & 86 & 86 & 86 \\
\hline
\end{tabular}

+ initial structure generated from cotranslational folding with torsional energy calculation 
++ optimized structure following the folding path determined by the torsional propensity

Table 2. Similarity among Structures in Iterations

\begin{tabular}{|c|c|c|c|c|c|c|c|c|}
\hline & init. & opt. 1 & opt. 2 & opt. 3 & opt. 4 & opt. 5 & opt. 6 & exp. s. \\
\hline \multicolumn{9}{|l|}{ init.+ } \\
\hline $\log P r$ & -16.00 & -4.62 & -3.50 & -4.80 & -4.84 & -4.84 & -4.85 & -0.80 \\
\hline $\begin{array}{l}\text { RamRMSD } \\
\text { opt. } 1++\end{array}$ & 0.00 & 47.17 & 49.75 & 78.61 & 78.39 & 78.28 & 78.27 & 119.67 \\
\hline $\log P r$ & & -16.00 & -6.75 & -3.88 & -3.98 & -4.84 & -4.02 & -0.80 \\
\hline $\begin{array}{l}\text { RamRMSD } \\
\text { opt. } 2\end{array}$ & & 0.00 & 29.93 & 61.63 & 61.27 & 61.25 & 61.26 & 134.82 \\
\hline $\log P r$ & & & -16.00 & -7.02 & -5.29 & -5.25 & -5.23 & -1.01 \\
\hline $\begin{array}{l}\text { RamRMSD } \\
\text { opt. } 3\end{array}$ & & & 0.00 & 45.08 & 45.55 & 45.73 & 45.81 & 116.68 \\
\hline $\log P r$ & & & & -16.00 & -11.08 & -10.14 & -10.13 & -0.84 \\
\hline $\begin{array}{l}\text { RamRMSD } \\
\text { opt. } 4\end{array}$ & & & & 0.00 & 0.68 & 1.00 & 1.11 & 135.88 \\
\hline $\log P r$ & & & & & -16.00 & -13.50 & -13.47 & -0.82 \\
\hline $\begin{array}{l}\text { RamRMSD } \\
\text { opt. } 5\end{array}$ & & & & & 0.00 & 0.41 & 0.52 & 136.08 \\
\hline $\log P r$ & & & & & & -16.00 & -15.18 & -0.82 \\
\hline $\begin{array}{l}\text { RamRMSD } \\
\text { opt. } 6\end{array}$ & & & & & & 0.00 & 0.13 & 136.23 \\
\hline $\log P r$ & & & & & & & -16.00 & -0.82 \\
\hline $\begin{array}{l}\text { RamRMSD } \\
\text { exp. s.* }\end{array}$ & & & & & & & 0.00 & 136.28 \\
\hline $\log P r$ & & & & & & & & -16.00 \\
\hline RamRMSD & & & & & & & & 0.00 \\
\hline
\end{tabular}

+ initial structure generated from cotranslational torsional folding

++ optimized structure following the sequence of folding based on the torsional propensity

* experimentally determined structure

Figure 1. Torque between two residues.

The torsional inertia is determined from the length of the rotational radius and the mass of the object. The components of torque in the polypeptide chain are displayed.

Figure 2. Difference of torque among various bond angles.

The magnitude of the torque varies according to the angles of bending of the rotating from stalled residues. The torque is maximized as it turns orthogonally while it vanishes when it turns in parallel.

Figure 3. Dihedral angles of Torsional Bonds of Simulated Structures and Experimentally Determined Structure.

Dihedral angles of the most representative structure of model 21 of NMR experiment were shown with blue line. Dihedral angles of initial structure from the sequential search of torsional global energy minima following the concept of cotranslational folding were shown with orange line. Dihedral angles of final structure from the iterative optimization according to the search sequence base on torsional property were shown with yellow line. While experimental structure showed rather regular oscillation along the $0^{\underline{0}}$ line, simulated structures 
mainly showed positive angles in the N-terminal region. Though initial structure and final structure are similar, optimized structure showed strong deviation in the C-terminal region.

Figure 4. Change of Potential Energy during the Initializations and Optimization.

The change of potential energy during the initial structure generation and further iterative optimization is shown in blue line. The potential energy shows drastic fluctuations during the initialization of cotranslational folding which partly indicates that addition of amino acid is not always either favorable or unfavorable. This fluctuation is different from following iterative optimizations reflecting the strong effect of the change of configurations. During the optimization processes, the potential energy decreased with saltatory tendency. After three iterations of optimizations, the potential energy remained as being rather conserved indicating the fast convergence of the algorithm to the local energy minima.

Figure 5. Structure of 1n9v.

The 3D stick model of the structure of the angiotensin peptide(PDB entry 1n9v; "DRVYIHPF") is displayed. Loop structure was found among the most of the peptide(from $2^{\text {nd }}$ to $8^{\text {th }}$ residue) and drawn with blue cylinder. Carbon atoms are shown with gray color, nitrogen atoms with blue color, oxygen atoms with red color, and hydrogen atoms with white color. Some hydrogen atoms are shown as single spheres ignoring the bondage information.

Figure 1.

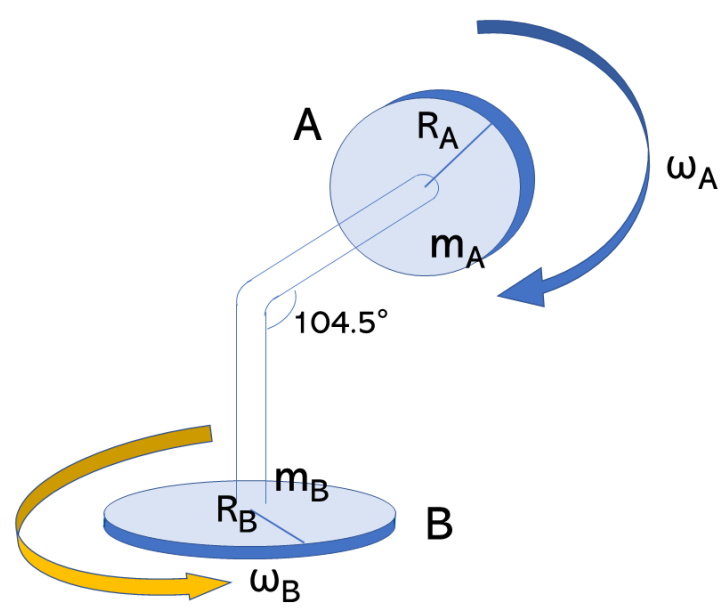

Figure 2 . 


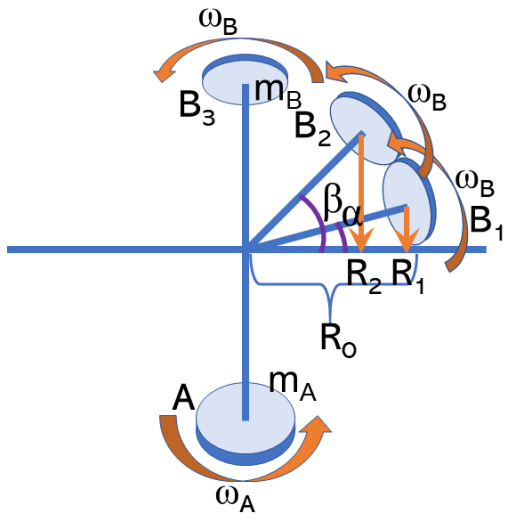

Figure 3.

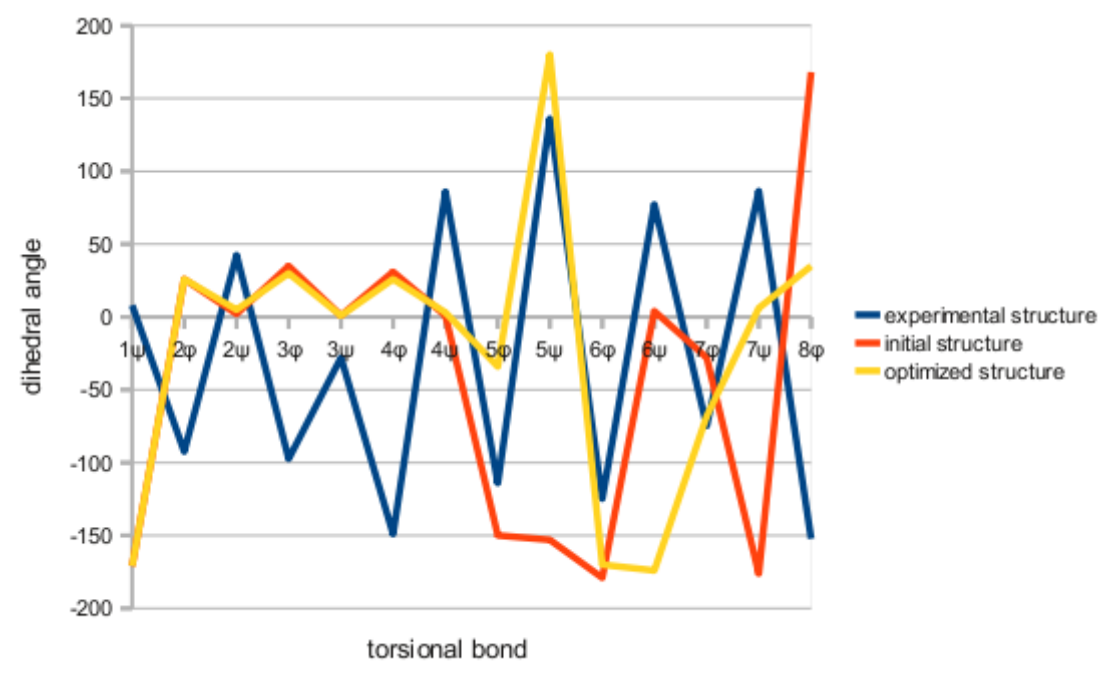




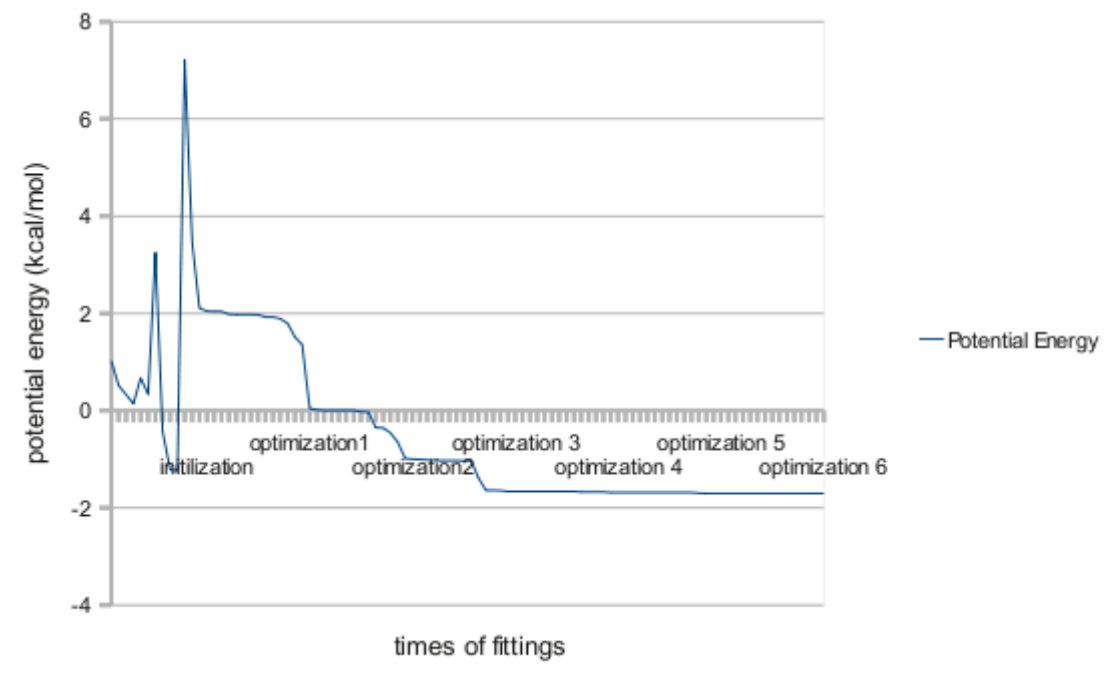

Figure 4.

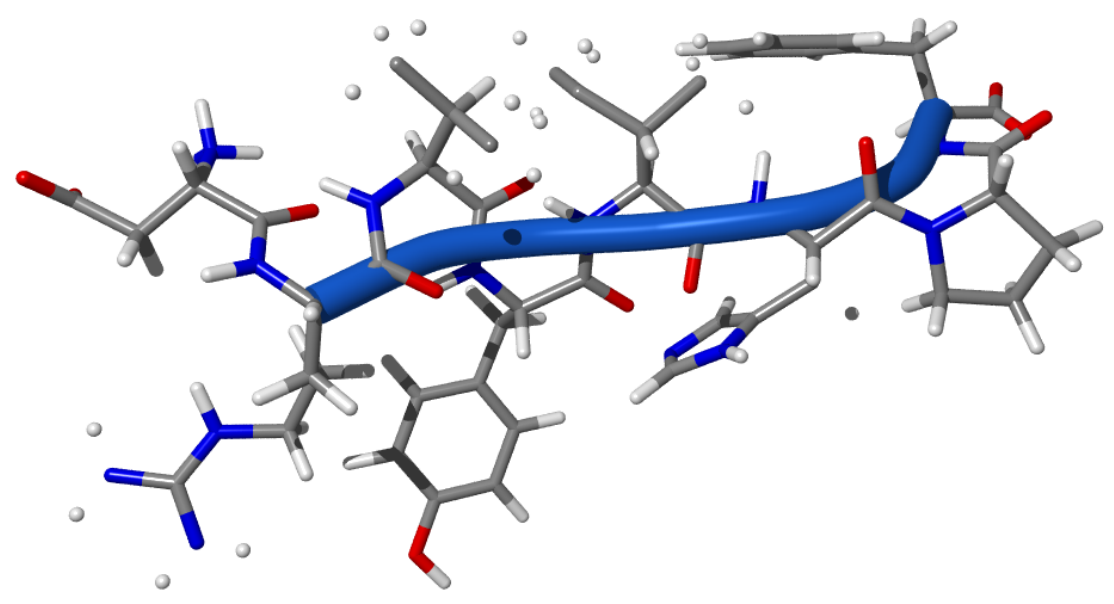

Figure 5 . 